\title{
Glucose Increases Interleukin-12 Gene Expression and Production in Stimulated Peripheral Blood Mononuclear Cells of Type 2 Diabetes Patients
}

\author{
Chien-Ming $\mathrm{Chu}^{1}$, Sheng-Fong $\mathrm{Kuo}^{2}$, Chung-Ching Hua ${ }^{1}$, Shao-Yun $\mathrm{Wu}^{3}$, Duen-Yau Chuang ${ }^{4}$, \\ Huang-Pin $\mathrm{Wu}^{1}$
}

Background: Lipopolysaccharide (LPS)-stimulated peripheral blood mononuclear cells (PBMCs) of type 2 diabetes patients produce more interleukin (IL)-12 under glucose treatment. The aim of this study was to determine whether increased IL-12 response in hyperglycemic LPS-stimulated PBMCs is due to increased gene expression or osmolarity.

Methods: $\quad$ LPS-stimulated PBMCs of 13 type 2 diabetes patients and 8 healthy controls were used for culture in the presence or absence of glucose or mannitol for $24 \mathrm{~h}$. The IL-12 gene expressions of PBMCs and IL-12 protein levels in supernatants were evaluated.

Results: $\quad$ After $24 \mathrm{~h}$, the stimulated PBMCs of diabetes patients expressed more IL-12 mRNA and produced more IL-12 protein following glucose treatment than those without glucose treatment and with mannitol treatment. Stimulated PBMCs of controls did not express more IL-12 mRNA and produce more IL-12 protein following glucose treatment than those without glucose treatment and with mannitol treatment.

Conclusions: Glucose increases the IL-12 production in stimulated PBMCs of diabetes patients through increased IL-12 gene

At a Glance Commentary

Scientific background of the subject

Lipopolysaccharide-stimulated peripheral blood mononuclear cells of type 2 diabetes patients treated with glucose produced more interleukin-12. Hyperglycemia also resulted in hyperosmolarity in blood. It is unclear whether the increased interleukin-12 production from diabetes patients with severe sepsis is due to enhanced gene expression or increased osmolarity.

\section{What this study adds to the field}

The increase in the glucose-mediated interleukin-12 production in diabetes patients was through an increase in the interleukin-12 gene expression. Hyperosmolarity did not influence interleukin-12 gene expression and production. Glucose might act as an enhancer of the lipopolysaccharide-related increase in IL-12 production. expression.

(Biomed J 2014;37:293-297)

Key words: hyperglycemia, interleukin-12, osmolarity, peripheral blood mononuclear cells, sepsis

A ntigen-presenting cells, such as monocytes and macrophages, are the primary source of interleukin (IL)-12. Importantly, IL-12 induces the polarization of $\mathrm{CD}^{+} \mathrm{T}$ cells to the $\mathrm{T}$ helper 1 (Th1) phenotype that mediates immunity against intracellular pathogens. ${ }^{[1]}$ Acute hyperglycemia influences the innate immune system. ${ }^{[2]}$ IL-12 responses from peripheral blood mononuclear cells (PBMCs) were influenced by glucose and insulin. ${ }^{[3]}$ The IL-12 production in the peripheral blood of high-risk insulin-dependent diabetes mellitus (DM) subjects was increased in vitro. ${ }^{[4]}$ Lipopolysaccharide (LPS)-stimulated PBMCs of type 2 DM patients treated with glucose produced more IL-12. ${ }^{[5]}$ Even though the hyperglycemic PBMCs were additionally treated with insulin, the PBMCs still produced more IL-12 in vitro.

From the ${ }^{1}$ Division of Pulmonary, Critical Care and Sleep Medicine, Chang Gung Memorial Hospital at Keelung, Chang Gung University College of Medicine, Taoyuan, Taiwan; ${ }^{2}$ Division of Metabolism and Endocrinology, Chang Gung Memorial Hospital at Keelung, Chang Gung University College of Medicine, Taoyuan, Taiwan; ${ }^{3}$ Nursing Department, Chang Gung Memorial Hospital at Keelung, Chang Gung University College of Medicine, Taoyuan, Taiwan; ${ }^{4}$ Department of Chemistry, National Chung-Hsing University, Taichung, Taiwan Received: Mar. 27, 2013; Accepted: Nov. 18, 2013

Correspondence to: Dr. Huang-Pin Wu, Division of Pulmonary, Critical Care and Sleep Medicine, Chang Gung Memorial Hospital at Keelung. 222, Maijin Rd, Anle District, Keelung 204, Taiwan (R.O.C.). Tel: 886-2-24313131 ext. 3173; Fax: 886-2-24335342; E-mail: whanpyng@cgmh.org.tw

DOI: $10.4103 / 2319-4170.132887$ 
Furthermore, elevated glucose levels and DM promoted the IL-12 gene expression in mouse macrophages. ${ }^{[6]} \mathrm{It}$ is reasonable to hypothesize that human hyperglycemic PBMCs produced more IL-12 through increased IL-12 gene expression.

Hyperglycemia also results in hyperosmolarity in blood. Compared with $5 \mathrm{mmol} / \mathrm{l}$ glucose, $15 \mathrm{mmol} / \mathrm{l} \mathrm{man-}$ nitol increased more IL- 6 and tumor necrosis factor (TNF)- $\alpha$ production from activated monocytes ${ }^{[7]}$ Higher osmolarities also resulted in increased secretion of IL-8, IL-6, IL-1 $\beta$, and TNF- $\alpha$ from epithelial cell lines. ${ }^{[8]} \mathrm{Up}$ to now, there is no report showing the association of high osmolarities with IL-12 production from cells.

It is unclear whether the increased IL-12 production is due to enhanced gene expression or increased osmolarity. Thus, we designed an in vitro experimental model to clarify the following: (1) whether glucose or hyperosmolarity affects the IL-12 gene expression in stimulated PBMCs and (2) whether glucose or hyperosmolarity enhances the IL-12 production from stimulated PBMCs.

\section{METHODS}

\section{Participants}

Between September 2009 and March 2010, 13 type 2 DM patients were enrolled. DM was defined according to the definition of the American Diabetes Association. ${ }^{[9]}$ Clinical data such as chest radiography, and white blood count and differential count were normal in all subjects. None of the subjects had pyuria and abnormal liver function test. For validating data, eight healthy controls with normal fasting blood glucose were enrolled from our healthy evaluation center. All subjects provided written informed consent. This study was approved by the Institutional Review Board at the Chang Gung Memorial Hospital.

\section{PBMCs preparation}

Whole blood $(20 \mathrm{ml})$ was obtained from each subject and immediately mixed with heparin at 0900-0950 hours. The PBMCs were isolated via differential centrifugation over Ficoll-Plaque (Amersham Biosciences, Uppsala, Sweden) within $2 \mathrm{~h}$ of collection.

\section{Cell culture}

We plated $5 \times 10^{5}$ PBMCs in 3 wells of a 24 -well culture plate (Nunclon, Aarhus, Denmark) in $1 \mathrm{ml}$ sterile tissue culture medium (RPMI 1640; Gibco, Grand Island, NY, USA) containing $5 \%$ heat-inactivated bovine serum, $200 \mathrm{mg} / \mathrm{dl}$ glucose, and $1 \mathrm{mM}$ sodium pyruvate (Gibco). The cells in the first well were stimulated with $1 \mu \mathrm{g} / \mathrm{ml}$ LPS (Sigma, St. Louis, MO, USA). The cells in the second well were stimulated with $1 \mu \mathrm{g} / \mathrm{ml}$ LPS and treated with additional $225 \mathrm{mg} / \mathrm{dl}(12.5 \mathrm{mM})$ glucose. The cells in the third well were stimulated with $1 \mu \mathrm{g} / \mathrm{ml}$ LPS and treated with $12.5 \mathrm{mM}$ mannitol. The culture plate was incubated at $37^{\circ} \mathrm{C}$ in $5 \% \mathrm{CO}_{2}$. Supernatants and PBMCs were sampled after $24 \mathrm{~h}$ of incubation. The PBMCs were homogenized in $1 \mathrm{ml}$ Trizol Reagent (Invitrogen Corp., Carlsbad, CA, USA). These samples were stored at $-80^{\circ} \mathrm{C}$ until use.

\section{Measurement of IL-12 levels}

The IL-12p40 concentration in the supernatants was measured with a human enzyme-linked immunosorbent assay (ELISA) kit (Becton Dickinson, Franklin Lakes, NJ, USA) according to the manufacturer's instruction. The minimum detectable concentration of this IL-12p40 ELISA kit was $3.9 \mathrm{pg} / \mathrm{ml}$.

\section{Detect IL-12p40 gene expression}

Total cellular RNA was extracted from the Trizol reagent with homogenized PBMCs according to the manufacturer's instruction. One microgram RNA was reverse transcribed into cDNA by the SuperScript Choice System for cDNA Synthesis Reagents (Promega Corp., Madison, WI, USA). For the detection of IL-12p40 gene expression, the cDNA was amplified using the following pair of primers: Forward primer 5'-GTG AGC CGT GAT TGT GC-3' and reverse primer 5'-CTG TGT CTT TTA GAG AGG TGG G-3'. The expression of a housekeeping gene, human acidic ribosomal protein (HuPO), was analyzed for normalizing the amount of cDNA in each sample. The primer pairs used in the polymerase chain reaction (PCR) were: Forward primer 5'-GCT TCC TGG AGG GTG TCC-3' and reverse primer 5'-GGA CTC GTT TGT ACC CGT TG-3'. The PCR mixture was amplified as follows: Activation step of $7 \mathrm{~min}$ at $94^{\circ} \mathrm{C}$, denaturation at $94^{\circ} \mathrm{C}$ for $1 \mathrm{~min}$, primer annealing at $62^{\circ} \mathrm{C}$ for $30 \mathrm{~s}$, and primer extension at $72^{\circ} \mathrm{C}$ for $1 \mathrm{~min}$ ( 28 cycles), followed by a final extension step for $10 \mathrm{~min}$ at $72^{\circ} \mathrm{C}$. The $28 \mathrm{PCR}$ cycles were chosen by a $26-38$ cycle profile [Figure 1]. The above primers were purchased from Purigo Biotech, Incorporation (Taipei, Taiwan). The PCR products were subjected to gel electrophoresis. Ethidium bromide staining and autography were used to detect two DNA bands. The DNA band of 137 bp corresponded to IL-12p40 cDNA and the other DNA band of $105 \mathrm{bp}$ corresponded to HuPO cDNA.

The PCR products were detected and analyzed on an image analyzer (Scientific Imaging Systems V.3.6.3., Kodak Company, Stamfort, CT, USA). The IL-12p40 mRNA expression was calculated as the ratio of IL-12 p40 amplicons per $10^{5} \mathrm{HuPO}$ PCR amplicons.

\section{Statistical analysis}

Statistical analysis was performed using the Statistical Package for Social Sciences (SPSS) V17.0 for Windows 
(SPSS, Inc., Chicago, IL, USA). Differences for categorical variables between two groups were compared using the Chi-Square test. Differences in continuous variables of two groups were analyzed by the Mann-Whitney test. Differences in continuous variables of the same patient were analyzed with the Wilcoxon signed ranks test. A value of $p<0.05$ was considered statistically significant.

\section{RESULTS}

\section{Effect of glucose and mannitol on IL-12 production from stimulated PBMCs}

The clinical characteristics in 13 type $2 \mathrm{DM}$ patients and healthy controls are shown in Table 1. Age in DM patients was higher than that in healthy controls. Stimulated PBMCs

Table 1: Clinical characteristics and cell analysis [median (min., max.) or number (percentage)]

\begin{tabular}{lccc}
\hline & $\begin{array}{c}\text { Diabetes } \\
\text { patients }(n=13)\end{array}$ & $\begin{array}{c}\text { Healthy controls } \\
(n=8)\end{array}$ & $p$ value \\
\hline $\begin{array}{l}\text { Age (years) } \\
\text { Gender }\end{array}$ & & & \\
Male & $6(46)$ & $6(75)$ & $0.00178 .0)$ \\
Female & $7(54)$ & $2(25)$ & \\
Glycohemoglobin & $7.8(6.8,11.0)$ & & \\
(HbA1c) (\%) & & & \\
Associated & & & \\
complications & & & \\
Peripheral arterial & $1(8)$ & & \\
occlusive disease & & & \\
Ischemic cerebral & $3(23)$ & & \\
arterial disease & & & \\
Coronary artery & $2(15)$ & & \\
disease & & & \\
Diabetic retinopathy & $6(46)$ & & \\
Diabetic nephropathy & $5(38)$ & & \\
Diabetic neuropathy & $1(8)$ & & \\
\hline
\end{tabular}

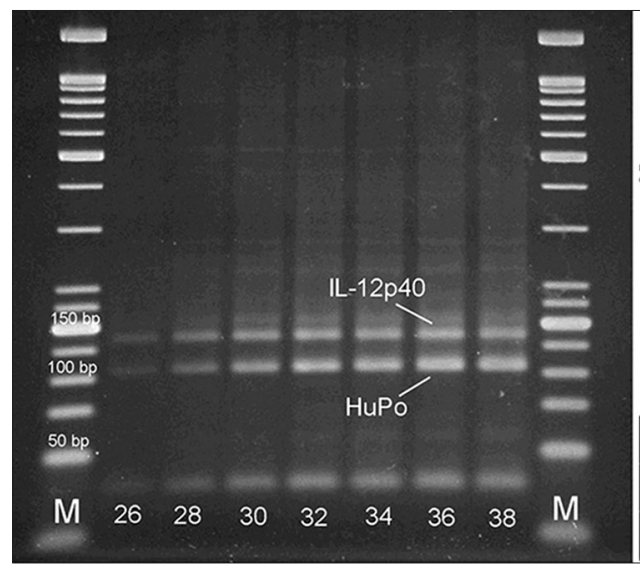

of DM patients produced more IL-12 following glucose treatment than those without glucose treatment and with mannitol treatment [Figure 2]. After $24 \mathrm{~h}$, no significant difference in the IL-12 production was observed among LPS-stimulated PBMCs of controls in the absence and presence of glucose or mannitol.

\section{Effect of glucose and mannitol on IL-12 mRNA expression from stimulated PBMCs}

Stimulated PBMCs of DM patients expressed more IL-12 mRNA following glucose treatment than those without glucose treatment and with mannitol treatment [Figure 3]. Stimulated PBMCs of controls did not express more IL-12 mRNA following glucose treatment than those without glucose treatment and with mannitol treatment.

\section{DISCUSSION}

This is the first study showing hyperglycemia-mediated enhanced IL-12 production by increased gene expression in stimulated PBMCs of type 2 diabetes patients. The increased production of IL-12 was not due to elevated blood osmolarity. The IL-12 protein could be detected in the supernatant of stimulated PBMCs after $24 \mathrm{~h}$ of culture. Moreover, the IL-12 protein production and gene expression were higher in the presence of glucose than in the absence of glucose or in the presence of mannitol. These results were in accordance with those of our previous study, which showed that glucose together with LPS might enhance production of the IL-12 protein. ${ }^{[5]}$ IL-12 proteins in the supernatants of cultured cells might also be from apoptosis cells. Hyperglycemic conditions enhanced both mouse and human thymocytes' survival. ${ }^{[10]}$ Thus, increased production of the IL-12 protein was not associated with the release from apoptosis cells.

The level of IL-12 mRNA expression was increased in LPS-stimulated PBMCs of DM patients with additional

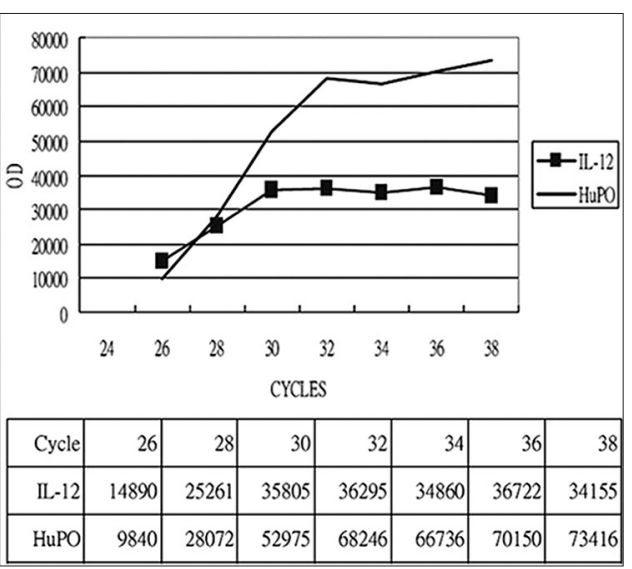

Figure 1: The interleukin (IL)-12p40 (137 bp) and human acidic ribosomal protein (HuPO) (105 bp) cDNAs from RNA isolated from lipopolysaccharide-stimulated peripheral blood mononuclear cells were amplified using the polymerase chain reaction (PCR) technique (M: Marker). Amount of PCR products over IL-12 and HuPO at different cycles is shown from one patient (OD: Optical density). 


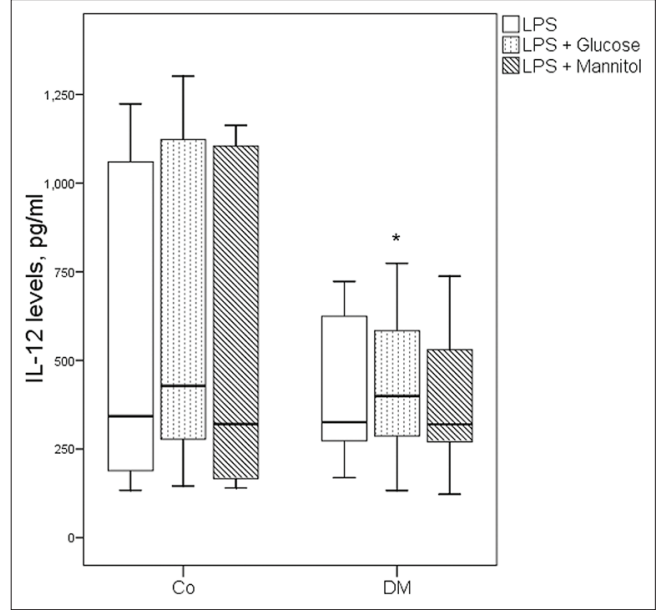

Figure 2: Interleukin (IL)-12 levels in the supernatants of lipopolysaccharide (LPS)-stimulated peripheral blood mononuclear cells with or without treatment of glucose or mannitol between diabetes mellitus (DM) patients and controls (Co). IL-12 levels were significantly increased after glucose treatment in DM patients but not in controls. Mannitol did not enhance IL-12 production either in DM patients or controls. $(* p<0.05$ compared with LPS and LPS + mannitol).

glucose treatment. IL-12 gene expression is mediated by nuclear factor-kappa B (NF- $\kappa \mathrm{B}){ }^{[11,12]}$ High glucose has been shown to induce NF- $\mathrm{\kappa B}$ activation. ${ }^{[13]}$ It is reasonable to hypothesize that increased IL-12 mRNA after glucose treatment was associated with NF- $\mathrm{KB}$ activation. PBMCs of DM patients transform glucose to some metabolites which might enhance IL-12 gene expression. Nonenzymatic glycoxidation of macromolecules ultimately resulting in the formation of advanced glycation endproduct (AGE) is enhanced in the presence of hyperglycemia, and systemic or local oxidant stress. ${ }^{[14]}$ Receptor of AGE has been identified as a central signal transduction receptor mediating long-lasting NF- $\mathrm{KB}$ activation in various cell types, including mononuclear phagocytes and vascular endothelium. ${ }^{[15]}$ This indicates that glucose may not directly enhance the IL-12 mRNA expression. The mediator of AGE may also explain the cause why IL-12 gene expression and production did not increase after glucose treatment in healthy controls. Simply, the PBMCs of healthy controls need more time to format AGE because the PBMCs of healthy controls usually are in euglycemia. This hypothesis needs further study for confirmation.

Our study further confirmed that mannitol did not increase more IL-12 gene expression in stimulated PBMCs of DM patients and healthy controls. This result is similar to that of Wen's study which showed that $15 \mathrm{mmol} / \mathrm{l}$ mannitol did not influence IL-12 gene expression in mouse macrophage.${ }^{[6]}$ More studies are needed to determine the influence of osmolarity on cytokine gene expression and production.

IL-12 response from PBMCs was restored in patients who survived severe sepsis. ${ }^{[16]}$ Impaired pre-operative mono-

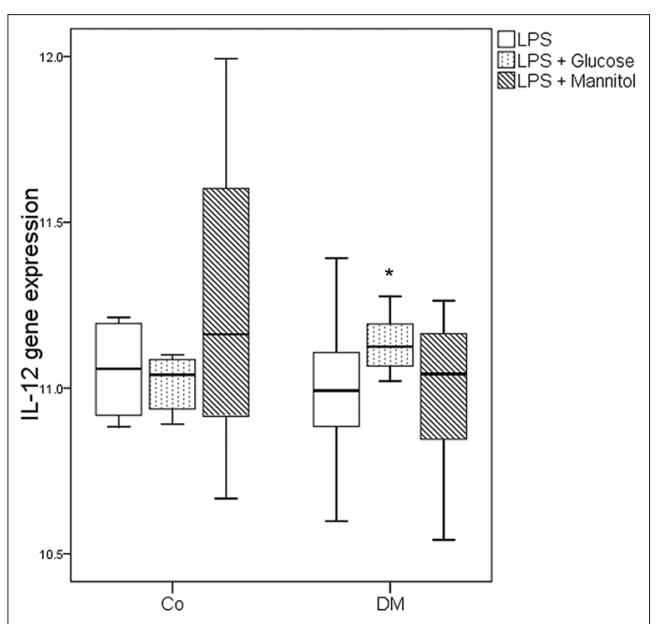

Figure 3: Log interleukin (IL)-12 gene expression in lipopolysaccharide (LPS)-stimulated peripheral blood mononuclear cells (PBMCs) between diabetes mellitus (DM) patients and controls (Co) with absence or presence of glucose or mannitol treatment. Stimulated PBMCs expressed more IL-12 mRNA following glucose treatment than those without glucose treatment and with mannitol treatment in DM patients but not in controls. (* $p<0.05$ compared with LPS and LPS + mannitol).

cyte IL-12 secretion was found in patients who developed fatal postoperative sepsis. ${ }^{[17]}$ These results are similar to those of Stanilova's study, ${ }^{[18]}$ which showed that survivors with severe sepsis produce more IL-12 from LPS-stimulated PBMCs than non-survivors. The main immunological function of IL-12 is to enhance native T lymphocyte differentiation to Th1 cells. Th1 cells secrete interferon- $\gamma$ that regulates macrophage and natural killer (NK) cell activation, stimulates immunoglobulin secretion by B cells, and enhances Th1 cell differentiation. Thus, increased IL-12 response in patients with severe sepsis may exert a protective effect by increased cellular immunity and phagocytic functions.

Macrovascular complication is important in DM patients. Inflammation is associated with the pathogenesis of coronary atherosclerosis. Circulating IL-12 may provide a mechanistic link between inflammation and Th1-type cytokine production in coronary atherosclerosis. ${ }^{[19]} \mathrm{IL}-12$ also induced T-cell recruitment into the atherosclerotic plaque. ${ }^{[20]}$ Furthermore, functional blockade of endogenous IL-12 by vaccination might result in a significant $68.5 \%$ reduction in atherogenesis in mice. Although hyperglycemia might increase IL-12 production in stimulated PBMCs of type 2 diabetes patients, ${ }^{[5]}$ overproduction of IL-12 during sepsis might harm the cardiovascular system.

When a gene is induced, the expression of its protein starts with the transcription of mRNA. The mRNA translocates to the rough endoplasmic reticulum (ER). After the protein is synthesized from mRNA by ribosomes and modified to become a matured protein in the ER, it is recruited into ER transport vesicles. The ER transport 
vesicles are transported from the ER to the trans-Golgi network, and then to constitutive secretory vesicles of the trans-Golgi network. The secretory vesicles of the protein require specific molecules, such as hormones or digestive enzymes, to regulate their exocytosis pathway. IL-12 was significantly produced from PBMCs $24 \mathrm{~h}$ after LPS stimulation and was not produced from unstimulated PBMCs. ${ }^{[5]}$ Thus, we can hypothesize that LPS is associated not only with IL-12 gene expression but also with IL-12 secretion. IL-12 protein exocytosis from intracellular space to extracellular space might be triggered by LPS. Intracellular IL-12 protein was digested if cells were not stimulated. In this study, the effect of glucose on IL-12 secretion was not determined because of no detection of intracellular IL-12 protein level. Whether glucose could regulate IL-12 exocytosis is unknown.

\section{Conclusions}

The increase in the glucose-mediated IL-12 production in stimulated PBMCs of type 2 DM patients was through an increase in the IL-12 gene expression. Hyperosmolarity did not influence IL-12 gene expression and production in type 2 DM patients. Glucose might act as an enhancer of the LPS-related increase in IL-12 production.

\section{Acknowledgments}

The authors would like to thank the Chang Gung Memorial Hospital for financially supporting this research in part under Contract No. CMRPG270421. The authors would also like to thank the clinical assistance from the Health Evaluation Center of the Chang Gung Memorial Hospital at Keelung.

\section{REFERENCES}

1. Watford WT, Moriguchi M, Morinobu A, O'Shea JJ. The biology of IL-12: Coordinating innate and adaptive immune responses. Cytokine Growth Factor Rev 2003;14:361-8.

2. Turina M, Fry DE, Polk HC. Acute hyperglycemia and the innate immune system: Clinical, cellular, and molecular aspects. Crit Care Med 2005;33:1624-33.

3. Wu HP, Chen CH, Hsieh HC, Liu YC. Effects of insulin and glucose on cytokine production from peripheral blood mononuclear cells. Chang Gung Med J 2008;31:253-9.

4. Szelachowska M, Kretowski A, Kinalska I. Increased in vitro interleukin-12 production by peripheral blood in high-risk IDDM first degree relatives. Horm Metab Res 1997;29:168-71.
5. Wu HP, Kuo SF, Wu SY, Chuang DY. High interleukin-12 production from stimulated peripheral blood mononuclear cells of type 2 diabetes patients. Cytokine 2010;51:298-304.

6. Wen Y, Gu J, Li SL, Reddy MA, Natarajan R, Nadler JL. Elevated glucose and diabetes promote interleukin-12 cytokine gene expression in mouse macrophages. Endocrinology 2006;147:2518-25.

7. Li-Bo Y, Wen-Bo Q, Xiao-Hong L, You-Lun F, Tie Z. Intermittent high glucose promotes expression of proinflammatory cytokines in monocytes. Inflamm Res 2011;60:367-70.

8. Schwartz L, Guais A, Pooya M, Abolhassani M. Is inflammation a consequence of extracellular hyperosmolarity? J Inflamm (Lond) 2009;6:21.

9. American Diabetes Association. Diagnosis and classification of diabetes mellitus. Diabetes Care 2006;29 Suppl 1:S43-8.

10. Ramakrishnan P, Kahn DA, Baltimore D. Anti-apoptotic effect of hyperglycemia can allow survival of potentially autoreactive $\mathrm{T}$ cells. Cell Death Differ 2011;18:690-9.

11. Song C, Zhang Q, Liu X, Shan Y. IL-12 and IL-10 Production are Differentially Regulated by Phosphatidylinositol 3-Kinase in Mast Cells. Scand J Immunol 2012;75:266-72.

12. Ellrichmann G, Thone J, Lee DH, Rupec RA, Gold R, Linker RA. Constitutive activity of NF-kappa B in myeloid cells drives pathogenicity of monocytes and macrophages during autoimmune neuroinflammation. J Neuroinflammation 2012;9:15.

13. Ha H, Yu MR, Choi YJ, Kitamura M, Lee HB. Role of high glucose-induced nuclear factor-kappaB activation in monocyte chemoattractant protein-1 expression by mesangial cells. J Am Soc Nephrol 2002;13:894-902.

14. Brownlee M. Advanced protein glycosylation in diabetes and aging. Annu Rev Med 1995;46:223-34.

15. Bierhaus A, Schiekofer S, Schwaninger M, Andrassy M, Humpert PM, Chen J, et al. Diabetes-associated sustained activation of the transcription factor nuclear factor-kappaB. Diabetes 2001;50:2792-808.

16. Wu HP, Shih CC, Lin CY, Hua CC, Chuang DY. Serial increase of IL-12 response and human leukocyte antigen-DR expression in severe sepsis survivors. Crit Care 2011;15:R224.

17. Weighardt H, Heidecke CD, Westerholt A, Emmanuilidis K, Maier S, Veit M, et al. Impaired monocyte IL-12 production before surgery as a predictive factor for the lethal outcome of postoperative sepsis. Ann Surg 2002;235:560-7.

18. Stanilova SA, Karakolev ZT, Dimov GS, Dobreva ZG, Miteva LD, Slavov ES, et al. High interleukin 12 and low interleukin 10 production after in vitro stimulation detected in sepsis survivors. Intensive Care Med 2005;31:401-7.

19. Ranjbaran H, Sokol SI, Gallo A, Eid RE, Iakimov AO, D“Alessio A, et al. An inflammatory pathway of IFN-gamma production in coronary atherosclerosis. J Immunol 2007;178:592-604.

20. Zhang X, Niessner A, Nakajima T, Ma-Krupa W, Kopecky SL, Frye RL, et al. Interleukin 12 induces T-cell recruitment into the atherosclerotic plaque. Circ Res 2006;98:524-31. 